\title{
Some closed-form solutions for buckling of straight beams with varying cross-section by Variational Iteration Method with Generalized Lagrange Multipliers
}

\author{
Ugurcan Eroglu ${ }^{a}$, Ekrem Tufekci ${ }^{b^{*}}$ \\ ${ }^{a, b}$ Istanbul Technical University, Faculty of Mechanical Engineering \\ ${ }^{*}$ E-mail address: ueroglu@itu.edu.tr ${ }^{\mathrm{a}}$, tufekcie@itu.edu.tr ${ }^{\mathrm{b}}$
}

Received date: 05.09 .2018

Accepted date: 02.10 .2018

ORCID numbers of authors

0000-0002-2446-0947 ${ }^{\mathrm{a}}, 0000-0003-3991-4005^{\mathrm{b}}$

\begin{abstract}
This study aims to derive approximate closed-form solutions for critical loads of straight beams with variable cross-section. The governing equations are derived for purely flexible beam for small displacements and rotation and turned into non-dimensional form. Approximate solutions to the set of equations for stability problems are searched by Variational Iteration Method with Generalized Lagrange Multipliers. It turns out that highly accurate approximate buckling loads for cantilever beams with constant or variable section can be obtained in closed-form. Many novel closed-form solutions for critical load of such structures, which may serve as benchmark solutions, are presented.
\end{abstract}

Keywords: beam theory, closed-form solutions, variational iteration method, buckling.

\section{Introduction}

Closed-form solutions are of practical importance to engineers and designers as they help to better understand the contributions of different physical parameters involved in a problem to the output, which may be static deflection, state of stress at a point, natural frequency, etc. This is important as it paves the way to essential elements of modern engineering, such as of optimum design and monitoring of structures. In addition, closed-form solutions may serve as benchmark solutions to numerical methods which are frequently used in modern time. Unfortunately, it is possible to obtain exact solutions in closed-form only for very special cases.

Not surprisingly, majority of closed-form solutions are presented in the literature for one dimensional structures [1], which is a reduced representation of 3-dimensional continua under reasonable assumptions and simplifications $[2,3]$. There are, of course, almost countless contributions on the field of mechanics of beam-like structures since the middle of $18^{\text {th }}$ century, but reviewing the entire bibliography would be out of the scope of this study. Rather, dedicated readers are kindly referred to the monographs by Love [3] and Timoshenko [4], to have a better insight about especially early works on this subject, which eventually formed the basis of structural engineering. 
Earliest investigation on the buckling of columns is due to Euler [5,6]. Without being exhaustive, one may quote Engesser [7], Dinnik [8], and Duncan [9] as other early contributors in the field. Moving on to the more recent investigations, one may quote contributions by Elishakoff and his co-workers [1, 10-14] concerning semi-inverse solutions for buckling of straight beams with continuously varying bending rigidity. These solutions make sense considering the introduction of functionally graded materials [15], and advances in their manufacturing [16]. Indeed, variation of bending rigidity along the beam axis may be due to smooth variation of cross-section, functional grading of the material, or both. Nevertheless, solution of a direct problem in closed-form, i.e. determination of critical load for a known material property and geometry, is still a challenging one. Yet, there are valuable contributions in the modern literature, such as the ones by Ruta et al. [17, 18], based on a onedimensional model for thin-walled beams [19], which provides exact solution to critical loads in closed-form, by Gupta et al. [20] for post-buckling behavior of laminated beams, Mercan and Civalek [21] for critical load of nanobeams, and Abbondanza et al. [22] for vibration frequencies and buckling loads of nanobeams. In addition, there are numerous studies which focus on numerical solutions of such problems, which ensures required accuracy for engineering applications when tackled the numerical problems, such as locking, but lack generality as they require the numerical values of the parameters of the problem. Instead, an approximate solution is aimed here. For this purpose, Variational Iteration Method (VIM), which has been shown to be a very simple and effective semi-analytical technique, is utilized. This method is developed by He [23-25], basically for solutions of non-linear problems. Reviews and more detailed explanations about the method can be seen in [26,27]. Since the initiation of the method, there have been many modifications and improvements introduced to it [28-32], for the reviews of which we refer to the note by He [33]. VIM is recently used for solutions of many different structural problems, see for example [34-38].

In this contribution, the aim is to present some approximate, yet accurate, solutions for buckling loads of straight beams of variable section. For this purpose, the system of equations is briefly derived and turned into non-dimensional form. As the solution technique, Variational Iteration Method with Generalized Lagrange Multiplier, which has been shown recently to be a very neat procedure for linear differential equation systems, is used. Amongst the classical boundary conditions, we focus on cantilevered beams as it is the only case that one can obtain real roots of the characteristic equation in closed-form. This may seem limiting, however, cantilevered beams of variable section may be an accurate model for many practical engineering problems. Moreover, being in closed-form, presented results may be used for benchmark purposes for different approximate and numerical techniques. To this aim, considering different variations of the cross-section, some new closed-form solutions for cantilevered beams are presented.

\section{Governing Equations}

Consider copies of a plane region, $\Re \subset \square^{2}$, attached orthogonally to a line of length $L, C_{0}$, through their centroids. The region occupied by this construction represents the reference configuration of the beam, $B^{0}$, for parameterization of which a Cartesian coordinate system, $\{x, y, z\}$, with base vectors $\{\mathbf{i}, \mathbf{j}, \mathbf{k}\}$ is introduced. With proper selection of an origin, it is assumed that $C_{0}$ is along $\mathbf{k}$, and, therefore, $\mathfrak{R}$ lies on coordinate plane $\{\mathbf{i}, \mathbf{j}\}$, which yields 
$B^{0}=\mathfrak{R} \times[0, L]$. Furthermore, principal axes of $\mathfrak{R}$ is assumed to be coincident with axes $x$ and $y$, for simplicity in constitutive modelling.

Current configuration of the beam, $B$, is described by regular vector field $\mathbf{r}(z)(=\mathbf{k}+\mathbf{u}(z))$ which represents the positions of each point, $C$, initially on $C_{0}$ and with coordinate $z$, and an orthogonal tensor $\mathbf{R}(z)$, providing the rotation of cross-sections, assumed to remain planar.

The expressions of finite deformation measures in current configuration $[17,18]$,

$$
\boldsymbol{\varepsilon}(z)=\frac{d}{d z}(z \mathbf{k}+\mathbf{u}(z))-\mathbf{R}(z) \mathbf{k}, \quad \chi(z)=\frac{d \mathbf{R}(z)}{d z} \mathbf{R}^{T}(z)
$$

where $\varepsilon(z)$ is the difference between the tangents of $C$ and $C_{0}$, pushed-forward from $B^{0}$ to $B$, and $\chi(z)$ presents the curvature of $C$.

The balance equations in actual configuration reads $[2,18]$,

$$
\frac{d \mathbf{F}(z)}{d z}+\mathbf{p}(z)=0, \frac{d \mathbf{M}(z)}{d z}+\frac{d}{d z}(z \mathbf{k}+\mathbf{u}(z)) \times \mathbf{F}(z)+\mathbf{m}(z)=0
$$

where $\mathbf{F}(z), \mathbf{M}(z), \mathbf{p}(z)$ and $\mathbf{m}(z)$ stand for internal force, internal couple, distributed external force and distributed external couple, respectively.

The analysis herein is limited such that the curve $C$ remains at $\{\mathbf{j}, \mathbf{k}\}$ plane. Then,

$$
\begin{gathered}
\mathbf{u}=u_{y} \mathbf{j}+u_{z} \mathbf{k}, \\
\mathbf{R}=\left(\begin{array}{ccc}
1 & 0 & 0 \\
0 & \cos \Omega_{x} & -\sin \Omega_{x} \\
0 & \sin \Omega_{x} & \cos \Omega_{x}
\end{array}\right), \\
\boldsymbol{\varepsilon}=\gamma_{y} \mathbf{j}+\varepsilon_{z} \mathbf{k}, \\
\chi=\chi_{x} \mathbf{j} \wedge \mathbf{k}, \\
\mathbf{F}=F_{y} \mathbf{j}+F_{z} \mathbf{k} \\
\mathbf{M}=M_{x} \mathbf{i},
\end{gathered}
$$

where the dependence of each field on $z$ is omitted for simplicity of the notation. 
Let us assume vanishing of the axial strain and shear strain, and a linear relation between the curvature of the deformed beam axis and the bending couple in the form,

$$
\varepsilon_{z}(z)=0, \quad \gamma_{y}(z)=0, \quad \chi_{x}(z)=\frac{M_{x}(z)}{E I_{x}(z)}
$$

where $E$ is the modulus of elasticity of the material and $I_{x}$ is the moment of inertia of the cross-sections about the axis $x$. The bending rigidity $E I_{x}(z)\left(=E I_{x 0} f(z)\right)$ is assumed to depend on the position along the axis, $z$, which may be due to variation of material properties, or smooth variation of cross-sectional dimensions, or both.

Let us further assume that $B^{0}$ is pre-loaded by what results an axial compressive force $N(z)$, which is assumed to be known and may be due to self-weight or an external action.

The system of differential equation for determination of configuration $B$ which is assumed to be in the neighborhood of $B^{0}$, thus linearized, by static perturbation technique $[17,18,39]$, reads,

$$
\begin{aligned}
& \frac{d \bar{u}_{z}}{d z}+\bar{\Omega}_{x}=0, \frac{d \bar{\Omega}_{x}}{d z}=\frac{\bar{M}_{x}}{E I_{x}} \\
& \frac{d \bar{F}_{y}}{d z}=0, \quad \frac{d \bar{M}_{x}}{d z}=\bar{F}_{y}-\bar{\Omega}_{x} N,
\end{aligned}
$$

where, superimposed bar denotes the first order derivative of indicated field with respect to an evolution parameter, hence the first order increments to the fields given in $(3)[17,18,39]$.

Equation system (5) is identical to that given in [40]. With the following non-dimensional quantities,

$$
\begin{aligned}
& Z=\frac{z}{L}, \quad T=\frac{\bar{F}_{y} L^{2}}{E I_{x 0}}, \quad P=\frac{N L^{2}}{E I_{x 0}} \\
& M=\frac{\bar{M}_{x} L}{E I_{x 0}}, \quad U=\frac{\bar{u}_{z}}{L}, \quad \theta=\bar{\Omega}_{x},
\end{aligned}
$$

System of equations given in (6) may be represented in matrix form as below. 


$$
\begin{aligned}
& \frac{d \mathbf{y}}{d Z}=\mathbf{A}(Z) \mathbf{y}, \quad \mathbf{y}=\left\{\begin{array}{llll}
U & \theta & T & M
\end{array}\right\}^{\mathrm{T}} \\
& \mathbf{A}(Z)=\left(\begin{array}{cccc}
0 & -1 & 0 & 0 \\
0 & 0 & 0 & \frac{1}{f(Z)} \\
0 & 0 & 0 & 0 \\
0 & -P & 1 & 0
\end{array}\right) .
\end{aligned}
$$

with solution,

$$
\mathbf{y}=\mathbf{Y}\left(Z, Z_{0}\right) \mathbf{y}\left(Z_{0}\right)
$$

where $\mathbf{Y}\left(Z, Z_{0}\right)$ is the matricant of system $(7)_{1}$ about an initial point $Z_{0}$, and $\mathbf{y}\left(Z_{0}\right)$ lists the initial values of field functions [41]. Note that when the coefficients matrix $\mathbf{A}$ consists of constant components, matricant of Eq. (7) ${ }_{1}$ is given by matrix exponential of $z \mathbf{A}$, which may be obtained exactly by Cayley-Hamilton theorem [42], or approximately by power series expansion. If the matrix $\mathbf{A}$ can be reduced into a triangular form, then again an exact solution may be found to Eq.(7) by successive integrations of the equations [43], similar to solution of an algebraic equation system by Gauss elimination method. Neither of these conditions hold in our case. Even in such situations, it might be possible to find an exact solution to the system of equations which requires a commutativity between $\mathbf{A}$ and matrix exponential of its integral. This is a very restrictive condition in practical point of view hence, search for approximate solution to Eq. (7) becomes inevitable.

\section{Variational Iteration Method}

A kind of VIM with a suitably modified Lagrange Multipliers for system of differential equations proposed by Altintan and Ugur [32] will be followed here. Even if the essence of the method is to tackle the nonlinear problems, here we will apply restricted variation to the part of matrix A which makes an exact solution to Eq.(7) impossible.

$$
\mathbf{y}_{k+1}(Z)=\mathbf{y}_{k}(Z)+\int_{Z_{0}}^{Z} \boldsymbol{\Lambda}(\zeta ; Z)\left(\frac{d \mathbf{y}_{k}(\zeta)}{d \zeta}-L_{1} \mathbf{y}_{k}(\zeta)-L_{2} \tilde{\mathbf{y}}_{k}(\zeta)\right) d \zeta
$$

where subscript $k$ denotes the order of approximation, and superimposed tilde denotes the variation of the indicated field is restricted. $L_{1}$ and $L_{2}$ are linear operators defined as below.

$$
L_{1} \mathbf{y}=\mathbf{A}_{1} \mathbf{y}, \quad L_{2} \mathbf{y}=\mathbf{A}_{2} \mathbf{y}
$$


where,

$$
\mathbf{A}_{1}=\left(\begin{array}{cccc}
0 & -1 & 0 & 0 \\
0 & 0 & 0 & \frac{1}{f(Z)} \\
0 & 0 & 0 & 0 \\
0 & 0 & 1 & 0
\end{array}\right), \mathbf{A}_{2}=\left(\begin{array}{cccc}
0 & 0 & 0 & 0 \\
0 & 0 & 0 & 0 \\
0 & 0 & 0 & 0 \\
0 & -P & 0 & 0
\end{array}\right) .
$$

With those definitions at hand, the so-called Generalized Lagrange Multiplier in Eq. (9), $\Lambda(\zeta ; Z)$ becomes [32],

$$
\boldsymbol{\Lambda}(\zeta ; Z)=-\boldsymbol{\Psi}(\zeta, Z)
$$

Where $\mathbf{\Psi}(\zeta, Z)$ is the matricant of system $d \mathbf{y} / d \zeta=\mathbf{A}_{1} \mathbf{y}$, about Z. Properties of matricant yields [32, 42],

$$
\boldsymbol{\Lambda}(\zeta ; Z)=\boldsymbol{\Psi}\left(Z, Z_{0}\right) \boldsymbol{\Psi}^{-1}\left(\zeta, Z_{0}\right)
$$

A recent contribution by Yildirim [44] provides the components of matricant, also known as fundamental matrix or transfer matrix, for constant cross-section.

If $(k+1)^{\text {th }}$ approximation of $\mathbf{y}$ is written similar to Eq. (8),

$$
\mathbf{y}_{k+1}(Z)=\mathbf{Y}_{k+1}\left(Z, Z_{0}\right) \mathbf{y}\left(Z_{0}\right)
$$

where, with the help of Eqs. $(7,12,13)$,

$$
\mathbf{Y}_{k+1}\left(Z, Z_{0}\right)=\mathbf{Y}_{k}\left(Z, Z_{0}\right)+\Psi\left(Z, Z_{0}\right) \int_{Z_{0}}^{Z} \boldsymbol{\Psi}^{-1}\left(\zeta, \zeta_{0}\right)\left[\frac{d \mathbf{Y}_{k}\left(\zeta, Z_{0}\right)}{d \zeta}-\mathbf{A}(\zeta)\right] d \zeta
$$

Once an initial approximation to the matricant, $\mathbf{Y}_{0}\left(Z, Z_{0}\right)$, is made, successive iterations provided in Eq. (15) will yield the approximate matricant of the system (7) 1 . Then, it is a matter of solving the initial values $\mathbf{y}\left(Z_{0}\right)$, or looking for mathematical requirements for a non-trivial solution of them. In our case the latter holds true. These conditions for classical boundary conditions are listed below. 


$$
\begin{aligned}
& \begin{array}{cl}
U(0)=0, \theta(0)=0, & \left|\begin{array}{ll}
Y_{33, k} & Y_{34, k} \\
Y_{43, k} & Y_{44, k}
\end{array}\right|=0 \\
T(1)=0, M(1)=0 .
\end{array} \\
& \begin{array}{ll}
U(0)=0, M(0)=0, & \left|\begin{array}{ll}
Y_{12, k} & Y_{13, k} \\
Y_{42, k} & Y_{43, k}
\end{array}\right|=0
\end{array} \\
& \begin{array}{ll}
U(0)=0, \theta(0)=0, & \left|\begin{array}{ll}
Y_{13, k} & Y_{14, k} \\
Y_{23, k} & Y_{24, k}
\end{array}\right|=0
\end{array}
\end{aligned}
$$

where $Y_{i j, k}$ denote the components of $k^{\text {th }}$ order of approximation to matricant at $i^{\text {th }}$ row and $j^{\text {th }}$ column. It turns out it is possible to find closed form solutions only for clamped-free column as that is the only case yielding a characteristic equation of third order with real roots.

In the next section, we will search for closed form expressions exploiting Eq. (15) for different variations of cross-section.

\section{Closed-Form Solutions}

As the initial approximation of the matricant, we will use the solution of (7) 1 for $\mathbf{A}_{2}=0$, that is, the elastic curve in the absence of pre-loads:

$$
\mathbf{Y}_{0}\left(Z, Z_{0}\right)=\boldsymbol{\Psi}\left(Z, Z_{0}\right)
$$

A suitable selection of the initial point is $Z_{0}=0$, for the simplicity of solutions. The explicit expressions of matricant components at each iteration are provided for specific variations of cross-section and pre-load in the following sub-sections.

\subsection{Polynomial Bending Rigidity}

Here we present some solutions bending rigidity given by a polynomial function specified as below.

$$
f(Z) \equiv\left(1-\alpha_{1} Z\right)\left(1-\alpha_{2} Z\right)^{3}
$$

This variation of the bending rigidity may be interpreted as that of a rectangular cross-section with linearly varying height and width, and has been considered recently in [45]. 
For such a variation of bending rigidity, components of the zeroth order of approximation to matricant are listed below.

$$
\begin{gathered}
Y_{11,0}=1, Y_{12,0}=-Z, \\
Y_{13,0}=-\frac{Z\left(\alpha_{1}-\alpha_{2}\right)\left(Z\left(\alpha_{1}+\alpha_{2}\right)-2\right)-2\left(\alpha_{1} Z-1\right)\left(\alpha_{2} Z-1\right) \ln \left(1-\alpha_{1} Z\right)+2\left(\alpha_{1} Z-1\right)\left(\alpha_{2} Z-1\right) \ln \left(1-\alpha_{2} Z\right)}{2\left(\alpha_{1}-\alpha_{2}\right)^{3}\left(\alpha_{2} Z-1\right)}, \\
Y_{14,0}=-\frac{Z\left(\alpha_{1}-\alpha_{2}\right)\left(\alpha_{1}\left(3 \alpha_{2} Z-2\right)-\alpha_{2}^{2} Z\right)-2 \alpha_{1}\left(\alpha_{1} Z-1\right)\left(\alpha_{2} Z-1\right) \ln \left(1-\alpha_{1} Z\right)+2 \alpha_{1}\left(\alpha_{1} Z-1\right)\left(\alpha_{2} Z-1\right) \ln \left(1-\alpha_{2} Z\right)}{2\left(\alpha_{1}-\alpha_{2}\right)^{3}\left(\alpha_{2} Z-1\right)}, \\
Y_{21,0}=0, Y_{22,0}=0, \\
Y_{24,0}=\frac{Z\left(\alpha_{1}-\alpha_{2}\right)\left(-2 \alpha_{1}+\alpha_{1} \alpha_{2} Z+\alpha_{2}^{2} Z\right)-2 \alpha_{1}\left(\alpha_{2} Z-1\right)^{2} \ln \left(1-\alpha_{1} Z\right)+2 \alpha_{1}\left(\alpha_{2} Z-1\right)^{2} \ln \left(1-\alpha_{2} Z\right)}{2\left(\alpha_{1}-\alpha_{2}\right)^{3}\left(\alpha_{2} Z-1\right)^{2}}, \\
Y_{31,0}=0, Y_{32,0}=0, Y_{33,0}=1, Y_{34,0}=0 \\
Y_{41,0}=0, Y_{42,0}=0, Y_{43,0}=Z, Y_{44,0}=1
\end{gathered}
$$

We list below the characteristic equations of different orders of approximations for clampedfree column with polynomial bending rigidity.

$k=1: \quad 2\left(\alpha_{2}-1\right)\left(\alpha_{1}-\alpha_{2}\right)^{3}+P\left(\left(\alpha_{1}-\alpha_{2}\right)\left(\alpha_{1}\left(2-3 \alpha_{2}\right)+\alpha_{2}^{2}\right)+2\left(\alpha_{1}-1\right) \alpha_{1}\left(\alpha_{2}-1\right)\left(\ln \left(1-\alpha_{1}\right)-\ln \left(1-\alpha_{2}\right)\right)\right)=0$

$$
\begin{array}{ll} 
& 12\left(\alpha_{2}-1\right)^{2}\left(\alpha_{1}-\alpha_{2}\right)^{5}+P\left[12\left(\alpha_{1}-1\right) \alpha_{1}\left(\alpha_{2}-1\right)^{2}\left(\alpha_{1}-\alpha_{2}\right)^{2}\left(\ln \left(1-\alpha_{1}\right)-\ln \left(1-\alpha_{2}\right)\right)\right. \\
k=2: \quad & \left.-6\left(\alpha_{2}-1\right)\left(\alpha_{1}-\alpha_{2}\right)^{3}\left(\alpha_{1}\left(3 \alpha_{2}-2\right)-\alpha_{2}^{2}\right)\right]+P^{2}\left[( \alpha _ { 1 } - \alpha _ { 2 } ) \left(\alpha_{1}^{2}\left(16 \alpha_{2}-15\right)-2 \alpha_{1}\left(\alpha_{2}\left(\alpha_{2}+9\right)-9\right)\right.\right. \\
& \left.\left.+\alpha_{2}\left(\left(9-2 \alpha_{2}\right) \alpha_{2}-6\right)\right)-6\left(\alpha_{1}-1\right)\left(\alpha_{2}-1\right)\left(2 \alpha_{1} \alpha_{2}+\left(\alpha_{1}-3\right) \alpha_{1}-\alpha_{2}^{2}+\alpha_{2}\right)\left(\ln \left(1-\alpha_{1}\right)-\ln \left(1-\alpha_{2}\right)\right)\right]=0
\end{array}
$$




$$
\begin{array}{ll} 
& 144\left(\alpha_{2}-1\right)^{3}\left(\alpha_{1}-\alpha_{2}\right)^{7}+P\left[144\left(\alpha_{1}-1\right) \alpha_{1}\left(\alpha_{2}-1\right)^{3}\left(\alpha_{1}-\alpha_{2}\right)^{4}\left(\ln \left(1-\alpha_{1}\right)-\log \left(1-\alpha_{2}\right)\right)\right. \\
& \left.-72\left(\alpha_{2}-1\right)^{2}\left(\alpha_{1}-\alpha_{2}\right)^{5}\left(\alpha_{1}\left(3 \alpha_{2}-2\right)-\alpha_{2}^{2}\right)\right]+P^{2}\left[1 2 ( \alpha _ { 2 } - 1 ) ( \alpha _ { 1 } - \alpha _ { 2 } ) ^ { 3 } \left(\alpha_{1}^{2}\left(16 \alpha_{2}-15\right)\right.\right. \\
k=3: & \left.-2 \alpha_{1}\left(\alpha_{2}\left(\alpha_{2}+9\right)-9\right)+\alpha_{2}\left(\left(9-2 \alpha_{2}\right) \alpha_{2}-6\right)\right)-72\left(\alpha_{1}-1\right)\left(\alpha_{2}-1\right)^{2}\left(\alpha_{1}-\alpha_{2}\right)^{2}\left(2 \alpha_{1} \alpha_{2}+\left(\alpha_{1}-3\right) \alpha_{1}\right. \\
& \left.\left.-\alpha_{2}^{2}+\alpha_{2}\right)\left(\ln \left(1-\alpha_{1}\right)-\ln \left(1-\alpha_{2}\right)\right)\right]+P^{3}\left[( \alpha _ { 2 } - \alpha _ { 1 } ) \left(\alpha_{1}^{3}\left(41 \alpha_{2}-40\right)+\alpha_{1}^{2}\left(\alpha_{2}\left(65 \alpha_{2}-224\right)+156\right)\right.\right. \\
& \left.+\alpha_{1}\left(\alpha_{2}\left(\left(28-43 \alpha_{2}\right) \alpha_{2}+138\right)-120\right)+\alpha_{2}\left(\alpha_{2}\left(\left(56-3 \alpha_{2}\right) \alpha_{2}-114\right)+60\right)\right)+12\left(\alpha_{1}-1\right)\left(\alpha_{2}-1\right)\left(\alpha_{1}^{3}\right. \\
& \left.\left.+\alpha_{1}^{2}\left(6 \alpha_{2}-8\right)+\alpha_{1}\left(10-9 \alpha_{2}\right)+\alpha_{2}\left(\left(7-2 \alpha_{2}\right) \alpha_{2}-5\right)\right)\left(\ln \left(1-\alpha_{1}\right)-\ln \left(1-\alpha_{2}\right)\right)\right]=0
\end{array}
$$

Solutions of Eqs.(20-22) provide the critical buckling loads at $k^{\text {th }}$ order, $P_{c r, k}$.

$$
\begin{aligned}
& P_{c r, 1}=-\frac{2\left(\alpha_{2}-1\right)\left(\alpha_{1}-\alpha_{2}\right)^{3}}{\left(\alpha_{1}-\alpha_{2}\right)\left(\alpha_{1}\left(2-3 \alpha_{2}\right)+\alpha_{2}^{2}\right)+2\left(\alpha_{1}-1\right) \alpha_{1}\left(\alpha_{2}-1\right) \ln \left(\frac{\alpha_{1}-1}{\alpha_{2}-1}\right)} \\
& \alpha_{1}=0 \Rightarrow P_{c r, 2}=\frac{\left(\alpha_{2}-1\right) \alpha_{2}^{2}\left(3 \alpha_{2}^{2}-\sqrt{3 \alpha_{2}\left(\alpha_{2}\left(\alpha_{2}\left(3 \alpha_{2}+8\right)-36\right)+24\right)+72\left(\alpha_{2}-1\right)^{2} \ln \left(1-\alpha_{2}\right)}\right)}{\alpha_{2}\left(\alpha_{2}\left(2 \alpha_{2}-9\right)+6\right)+6\left(\alpha_{2}-1\right)^{2} \ln \left(1-\alpha_{2}\right)} \\
& \alpha_{2}=0 \Rightarrow P_{c r, 2}=\frac{2 \alpha_{1}^{2}}{\sqrt{\left(\alpha_{1}-1\right)\left(6 \alpha_{1}+\ln \left(1-\alpha_{1}\right)\left(-4 \alpha_{1}+\left(\alpha_{1}-1\right) \ln \left(1-\alpha_{1}\right)+6\right)\right)}+\alpha_{1}-\left(\alpha_{1}-1\right) \ln \left(1-\alpha_{1}\right)} \\
& \alpha_{1}=0 \Rightarrow P_{c r, 3}=\frac{2}{c_{1}}\left[-\frac{2\left(\alpha_{2}-1\right)^{2} \alpha_{2}^{4}}{\sqrt[3]{c_{2}}}\left(12 \alpha_{2}\left(2 \alpha_{2}^{2}+3 \alpha_{2}-12\right)\left(\alpha_{2}-1\right)^{2} \ln \left(1-\alpha_{2}\right)-72\left(\alpha_{2}-1\right)^{4} \ln ^{2}\left(1-\alpha_{2}\right)\right.\right. \\
& \left.+\alpha_{2}^{2}\left(\alpha_{2}^{4}-96 \alpha_{2}^{3}+132 \alpha_{2}^{2}+36 \alpha_{2}-72\right)\right)+\sqrt[3]{c_{2}}+2\left(\alpha_{2}-1\right) \alpha_{2}^{2}\left(\alpha_{2}\left(2 \alpha_{2}^{2}-9 \alpha_{2}+6\right)\right. \\
& \left.\left.+6\left(\alpha_{2}-1\right)^{2} \ln \left(1-\alpha_{2}\right)\right)\right] \\
& \alpha_{2}=0 \Rightarrow P_{c r, 3}=\frac{1}{c_{3}}\left[-\frac{3 \alpha_{1}^{4}}{\sqrt[3]{c_{4}}}\left(\left(-35 \alpha_{1}^{2}+24 \alpha_{1}+12\right) \alpha_{1}^{2}+8\left(\alpha_{1}^{3}-\alpha_{1}^{2}-3 \alpha_{1}+3\right) \alpha_{1} \ln \left(1-\alpha_{1}\right)\right.\right. \\
& \left.-12\left(\alpha_{1}-1\right)^{2}\left(2 \alpha_{1}-1\right) \ln ^{2}\left(1-\alpha_{1}\right)\right)+\sqrt[3]{c_{4}}-3 \alpha_{1}^{2}\left(\left(6-5 \alpha_{1}\right) \alpha_{1}\right. \\
& \left.\left.+2\left(\alpha_{1}^{2}-4 \alpha_{1}+3\right) \ln \left(1-\alpha_{1}\right)\right)\right] \\
& c_{1}=\alpha_{2}\left(3 \alpha_{2}^{3}-56 \alpha_{2}^{2}+114 \alpha_{2}-60\right)+12\left(2 \alpha_{2}-5\right)\left(\alpha_{2}-1\right)^{2} \ln \left(1-\alpha_{2}\right)
\end{aligned}
$$




$$
\begin{aligned}
& c_{2}=-36 \alpha_{2}^{7}\left(17 \alpha_{2}^{5}-156 \alpha_{2}^{4}-498 \alpha_{2}^{3}+3120 \alpha_{2}^{2}-4284 \alpha_{2}+1800\right)\left(\alpha_{2}-1\right)^{5} \ln \left(1-\alpha_{2}\right)+650484 \alpha_{2}^{10} \\
& -432 \alpha_{2}^{6}\left(2 \alpha_{2}^{3}+15 \alpha_{2}^{2}-72 \alpha_{2}+75\right)\left(\alpha_{2}-1\right)^{7} \ln ^{2}\left(1-\alpha_{2}\right)+32400 \alpha_{2}^{8}-222048 \alpha_{2}^{9}+1728 \alpha_{2}^{6}\left(\alpha_{2}-1\right)^{9} \ln ^{3}\left(1-\alpha_{2}\right) \\
& -1052676 \alpha_{2}^{11}+1008324 \alpha_{2}^{12}-557424 \alpha_{2}^{13}+146079 \alpha_{2}^{14}+4031 \alpha_{2}^{15}-10815 \alpha_{2}^{16}+1689 \alpha_{2}^{17}-44 \alpha_{2}^{18} \\
& +3\left[\left(-432\left(\alpha_{2}^{4}-4 \alpha_{2}^{3}-18 \alpha_{2}^{2}+84 \alpha_{2}-75\right)\left(\alpha_{2}-1\right)^{4} \ln ^{2}\left(1-\alpha_{2}\right)-3456\left(\alpha_{2}-1\right)^{6} \ln ^{3}\left(1-\alpha_{2}\right)\right.\right. \\
& +72 \alpha_{2}\left(4 \alpha_{2}^{6}+15 \alpha_{2}^{5}-186 \alpha_{2}^{4}-117 \alpha_{2}^{3}+1596 \alpha_{2}^{2}-2214 \alpha_{2}+900\right)\left(\alpha_{2}-1\right)^{2} \ln \left(1-\alpha_{2}\right) \\
& +\alpha_{2}^{2}\left(24 \alpha_{2}^{8}-824 \alpha_{2}^{7}-1719 \alpha_{2}^{6}+14904 \alpha_{2}^{5}+2628 \alpha_{2}^{4}-100872 \alpha_{2}^{3}+180036 \alpha_{2}^{2}\right. \\
& \left.\left.\left.-126576 \alpha_{2}+32400\right)\right)\left(\alpha_{2}-1\right)^{6} \alpha_{2}^{12}\left(\alpha_{2}\left(3 \alpha_{2}^{3}-56 \alpha_{2}^{2}+114 \alpha_{2}-60\right)+12\left(2 \alpha_{2}-5\right)\left(\alpha_{2}-1\right)^{2} \ln \left(1-\alpha_{2}\right)\right)^{2}\right]^{1 / 2} \\
& \quad c_{3}=\alpha_{1}\left(-10 \alpha_{1}^{2}+39 \alpha_{1}-30\right)+3\left(\alpha_{1}^{3}-9 \alpha_{1}^{2}+18 \alpha_{1}-10\right) \ln \left(1-\alpha_{1}\right) \\
& \quad c_{4}=3\left[-375 \alpha_{1}^{12}+5220 \alpha_{1}^{11}-14778 \alpha_{1}^{10}+15336 \alpha_{1}^{9}-5400 \alpha_{1}^{8}+36\left(\alpha_{1}-1\right)^{3}\left(\alpha_{1}^{3}-15 \alpha_{1}^{2}\right.\right. \\
& \left.+48 \alpha_{1}-36\right) \alpha_{1}^{6} \ln ^{3}\left(1-\alpha_{1}\right)-36\left(\alpha_{1}-1\right)^{2}\left(7 \alpha_{1}^{4}-87 \alpha_{1}^{3}+279 \alpha_{1}^{2}-348 \alpha_{1}+150\right) \alpha_{1}^{6} \ln ^{2}\left(1-\alpha_{1}\right) \\
& \quad+36\left(15 \alpha_{1}^{5}-197 \alpha_{1}^{4}+786 \alpha_{1}^{3}-1342 \alpha_{1}^{2}+1038 \alpha_{1}-300\right) \alpha_{1}^{7} \ln \left(1-\alpha_{1}\right)+2 \sqrt{3}\left(\alpha _ { 1 } ^ { 1 2 } \left(\alpha _ { 1 } \left(-10 \alpha_{1}^{2}\right.\right.\right. \\
& \left.\left.+39 \alpha_{1}-30\right)+3\left(\alpha_{1}^{3}-9 \alpha_{1}^{2}+18 \alpha_{1}-10\right) \ln \left(1-\alpha_{1}\right)\right)^{2}\left(-8\left(11 \alpha_{1}^{3}-141 \alpha_{1}^{2}+294 \alpha_{1}-162\right)\left(\alpha_{1}-1\right)^{3} \ln ^{3}\left(1-\alpha_{1}\right)\right. \\
& +\alpha_{1}^{2}\left(10 \alpha_{1}^{4}-2964 \alpha_{1}^{3}+8571 \alpha_{1}^{2}-8316 \alpha_{1}+2700\right)+12\left(\alpha_{1}-13\right)\left(\alpha_{1}-1\right)^{5} \ln ^{4}\left(1-\alpha_{1}\right)+12\left(19 \alpha_{1}^{4}-288 \alpha_{1}^{3}\right. \\
& \left.+726 \alpha_{1}^{2}-684 \alpha_{1}+225\right)\left(\alpha_{1}-1\right)^{2} \ln ^{2}\left(1-\alpha_{1}\right)-6 \alpha_{1} \ln \left(1-\alpha_{1}\right)\left(43 \alpha_{1}^{5}-848 \alpha_{1}^{4}+3207 \alpha_{1}^{3}-4940 \alpha_{1}^{2}\right. \\
& \left.\left.\left.\left.+3438 \alpha_{1}-900\right)\right)\right)^{1 / 2}\right]
\end{aligned}
$$

Note that Eqs.(23-27) are the lowest roots of the characteristic equations for $1>\alpha_{1}, \alpha_{2} \geq 0$.

Table 1. Critical loads for variable height $\left(\alpha_{1}=0\right)$

\begin{tabular}{|c||c|c|c||c||}
\hline \multirow{2}{*}{$\alpha_{2}$} & \multicolumn{3}{|c||}{ Present } & [45] \\
\cline { 2 - 5 } & $P_{c r, 1}$ & $P_{c r, 2}$ & $P_{c r, 3}$ & 2.467 \\
\hline 0 & 2 & 2.536 & 2.465 & 2.023 \\
\hline 0.2 & 1.6 & 2.101 & 2.023 & 1.569 \\
\hline 0.4 & 1.2 & 1.660 & 1.565 & 1.098 \\
\hline 0.6 & 0.8 & 1.218 & 1.093 & 0.597 \\
\hline 0.8 & 0.4 & - & 0.588 & \\
\hline
\end{tabular}

Numerical results are given for constant width, variable height and constant height, variable width, in Table 1 and Table 2, respectively, from which one may see their convergence and accuracy. Very simple first order approximations of the critical loads seem to be impractical, while third order solutions are very accurate. The simplicity versus the relatively low accuracy of second order approximations may be debated; but the effects of geometrical parameters on the critical load are well represented. On the other hand, solution of second 
order approximation yields an imaginary root for $\alpha_{1}=0, \alpha_{2}=0.8$, which is possibly due to inability of the approximate displacement function to represent the actual mode shape.

Table 2. Critical loads for variable width $\left(\alpha_{2}=0\right)$

\begin{tabular}{|c|c|c|c||c|}
\hline \hline \multirow{2}{*}{$\alpha_{1}$} & \multicolumn{3}{|c|}{ Present } & \multirow{2}{*}{ [45] } \\
\cline { 2 - 4 } & $P_{c r, 1}$ & $P_{c r, 2}$ & $P_{c r, 3}$ & 2.467 \\
\hline \hline 0 & 2 & 2.536 & 2.465 & 2.316 \\
\hline 0.2 & 1.862 & 2.387 & 2.314 & 2.151 \\
\hline 0.4 & 1.711 & 2.227 & 2.148 & 1.968 \\
\hline 0.6 & 1.542 & 2.052 & 1.964 & 1.752 \\
\hline 0.8 & 1.339 & 1.855 & 1.747 & \\
\hline
\end{tabular}

In case of a square cross-section, i.e. $\alpha_{1}=\alpha_{2}=\alpha$,

$$
\begin{gathered}
P_{c r, 1}=-\frac{6(-1+\alpha)^{2}}{-3+2 \alpha} \\
P_{c r, 2}=\frac{10 \alpha(\alpha(2 \alpha-7)+8)+2 \sqrt{5} \sqrt{(\alpha-1)^{4}(4 \alpha(5 \alpha-9)+15)}-30}{4 \alpha-5} \\
P_{c r, 3}=\frac{2(-1+\alpha)^{2}\left(-128.076-35 c_{5}^{1 / 3}+1.913 c_{5}{ }^{2 / 3}+146.372 \alpha+28 c_{5}{ }^{1 / 3} \alpha-29.274 \alpha^{2}\right)}{c_{5}{ }^{1 / 3}(-7+6 \alpha)} \\
c_{5}=\left(6360 \alpha^{2}-1904 \alpha^{3}+7\left(385+\sqrt{10} \sqrt{15435-54180 \alpha+72600 \alpha^{2}-43904 \alpha^{3}+10080 \alpha^{4}}\right)\right. \\
\left.-6 \alpha\left(1190+\sqrt{10} \sqrt{15435-54180 \alpha+72600 \alpha^{2}-43904 \alpha^{3}+10080 \alpha^{4}}\right)\right)
\end{gathered}
$$

Table 3. Critical loads of column with square cross-section $\left(\alpha_{1}=\alpha_{2}=\alpha\right)$

\begin{tabular}{|c||c|c|c||c||}
\hline \hline \multirow{2}{*}{$\alpha$} & \multicolumn{3}{|c||}{ Present } & [45] \\
\cline { 2 - 4 } & $P_{c r, 1}$ & $P_{c r, 2}$ & $P_{c r, 3}$ & 2.467 \\
\hline \hline 0 & 2 & 2.536 & 2.465 & 1.884 \\
\hline 0.2 & 1.477 & 1.963 & 1.880 & 1.309 \\
\hline 0.4 & 0.982 & 1.406 & 1.304 & 0.757 \\
\hline 0.6 & 0.533 & 0.894 & 0.750 & 0.265 \\
\hline 0.8 & 0.171 & - & 0.259 & \\
\hline
\end{tabular}

Critical loads of different orders of approximations for variable square section is presented in Table 3. The outlook is very similar to first two tables: first order solutions are not accurate while the third order solutions are in a very good agreement with the literature. Second order solution in case of a very sharp change in cross-section results an imaginary root, again possibly due to inadequate prediction of the mode shape. This situation may be seen as a 
drawback but the use of classical beam theories for structures with rapid change of section can also be debated.

\subsection{Exponential Bending Rigidity}

Here the bending rigidity is assumed to vary exponentially, as it is common in the literature $[34,46]$.

$$
f(Z) \equiv e^{-\alpha Z}
$$

For such a variation of bending rigidity, components of the zeroth order of approximation to matricant are listed below.

$$
\begin{gathered}
Y_{11,0}=1, \quad Y_{12,0}=-Z, Y_{13,0}=\frac{\alpha(-Z)+e^{\alpha Z}(2-\alpha Z)-2}{\alpha^{3}}, \quad Y_{14,0}=\frac{\alpha Z-e^{\alpha Z}+1}{\alpha^{2}} \\
Y_{22,0}=1, \quad Y_{23,0}=\frac{e^{\alpha Z}(\alpha Z-1)+1}{\alpha^{2}}, \quad Y_{24,0}=\frac{e^{\alpha Z}-1}{\alpha} \\
Y_{33,0}=1, \quad Y_{43,0}=Z, \quad Y_{44,0}=1
\end{gathered}
$$

We list below the characteristic equations of different orders of approximations for clampedfree column with exponential bending rigidity.

$$
\begin{gathered}
k=1: \quad \alpha^{2}+\left(\alpha-e^{\alpha}+1\right) P=0 \\
k=2: \quad\left(-4 e^{\alpha}(\alpha-1)+e^{2 \alpha}-2 \alpha-5\right) P^{2}+4\left(\alpha-e^{\alpha}+1\right) \alpha^{2} P+4 \alpha^{4}=0 \\
k=3: \quad \begin{array}{l}
\left(9 e^{2 \alpha}(\alpha-2)-e^{3 \alpha}+3 \alpha+9 e^{\alpha}(2 \alpha+1)+10\right) P^{3} \\
-9\left(4 e^{\alpha}(\alpha-1)-e^{2 \alpha}+2 \alpha+5\right) \alpha^{2} P^{2}+36\left(\alpha-e^{\alpha}+1\right) \alpha^{4} P+36 \alpha^{6}=0
\end{array}
\end{gathered}
$$

Solutions of Eqs.(38-40) provide the critical buckling loads at $k^{\text {th }}$ order, $P_{c r, k}$.

$$
\begin{gathered}
P_{c r, 1}=\frac{\alpha^{2}}{-1+e^{\alpha}-\alpha} \\
P_{c r, 2}=-\frac{2 \alpha^{2}}{\left(1-e^{\alpha}+\alpha\right)-\sqrt{\left(6+2 e^{\alpha}(-3+\alpha)+\alpha(4+\alpha)\right)}}
\end{gathered}
$$




$$
\begin{aligned}
P_{c r, 3}=\frac{\alpha^{2}}{c_{6} \sqrt[3]{c_{7}}} & {\left[\sqrt[3]{3} c_{7}^{2 / 3}-3^{2 / 3}\left(-6 e^{2 \alpha}\left(2 \alpha^{2}+21\right)+4 e^{\alpha}\left(6 \alpha^{2}+6 \alpha+29\right)+e^{3 \alpha}(44-16 \alpha)\right.\right.} \\
+ & \left.\left.e^{4 \alpha}-8 \alpha-35\right)-3 \sqrt[3]{c_{7}}\left(-4 e^{\alpha}(\alpha-1)+e^{2 \alpha}-2 \alpha-5\right)\right]
\end{aligned}
$$

where,

$$
\begin{gathered}
c_{6}=\left(9 e^{2 \alpha}(\alpha-2)-e^{3 \alpha}+3 \alpha+9 e^{\alpha}(2 \alpha+1)+10\right) \\
c_{7}=-3\left(12 \alpha^{3}+84 \alpha^{2}+3 e^{4 \alpha}\left(4 \alpha^{2}-68 \alpha+165\right)+4 e^{3 \alpha}\left(6 \alpha^{3}+69 \alpha^{2}-396 \alpha+77\right)\right. \\
+6 e^{\alpha}\left(24 \alpha^{3}+118 \alpha^{2}+214 \alpha+165\right)+3 e^{2 \alpha}\left(84 \alpha^{3}+240 \alpha^{2}+104 \alpha-633\right)+180 \alpha \\
\left.-e^{6 \alpha}+6 e^{5 \alpha}(2 \alpha-3)+125\right)-2 \sqrt{3}\left(9 e^{2 \alpha}(\alpha-2)-e^{3 \alpha}+3 \alpha+9 e^{\alpha}(2 \alpha+1)+10\right)\left(-12 e^{5 \alpha}(\alpha-2)\right. \\
-6 e^{4 \alpha}\left(4 \alpha^{2}-25 \alpha+52\right)-8 e^{3 \alpha}\left(2 \alpha^{3}-30 \alpha^{2}+201 \alpha-434\right)+3 e^{2 \alpha}\left(120 \alpha^{3}-96 \alpha^{2}-776 \alpha-2033\right) \\
\left.+2\left(6 \alpha^{4}+44 \alpha^{3}+114 \alpha^{2}+129 \alpha+5\right)+12 e^{\alpha}\left(12 \alpha^{4}+72 \alpha^{3}+212 \alpha^{2}+295 \alpha+242\right)+e^{6 \alpha}\right)^{1 / 2}
\end{gathered}
$$

The numerical results for different geometries with comparison are given in Table 4. Very similar to the previous problem, convergence of the numerical results with the order of approximations are apparent. Also, agreement of the results with the literature are encouraging.

Table 4. Critical loads of clamped-free column with exponentially varying bending rigidity

\begin{tabular}{|c|c|c|c||c||}
\hline \multirow{2}{*}{$\alpha$} & \multicolumn{3}{|c|}{ Present } & \multirow{2}{*}{ [46] } \\
\cline { 2 - 5 } & $P_{c r, 1}$ & $P_{c r, 2}$ & $P_{c r, 3}$ & 2.467 \\
\hline \hline 0 & 2 & 2.536 & 2.465 & 2.394 \\
\hline 0.1 & 1.934 & 2.464 & 2.392 & 2.110 \\
\hline 0.5 & 1.681 & 2.187 & 2.109 & 1.782 \\
\hline 1.0 & 1.392 & 1.861 & 1.778 & 1.480 \\
\hline 1.5 & 1.135 & 1.531 & 1.476 & 1.209 \\
\hline 2.0 & 0.911 & 1.294 & 1.205 & \\
\hline
\end{tabular}

The essence and practical importance of the results provided herein is evident from the very good agreement of the numerical results with the existing literature. It must be noted that this approach to the solution of critical buckling load of columns provides very accurate closedform solutions by very simple integrations and determination of roots of polynomial equations. Classical VIM approaches to this problem may require dealing with heavy integrations, and consideration of higher order of approximations for convergence, which inevitably require numerical solution techniques to solve the characteristic equation. Indeed, it is reported in [34], for a very similar problem, that nine iterations are conducted, and series expansions of the variations of bending rigidity up to nine terms are used to obtain the 
numerical results. Even though their results are almost overlap with the exact solutions, the heavy integrations and computational cost must be taken into consideration.

\section{Conclusions}

This paper aims to derive some closed-form solutions for critical loads of columns with variable section. To this aim, Variational Iteration Method, modified for the system of linear differential equations, is utilized. It is found that the solutions to the approximate characteristic equations of up to third order are highly accurate for cantilevered beams, while other boundary conditions require the consideration of higher order approximations. Hence, some approximate closed-form solutions are presented, for the first time, for cantilevered columns of variable cross-section. The accuracy and versatility of the solution procedure are demonstrated by comparing the results presented in the literature, and a very good agreement is observed. The closed-form solutions presented herein, therefore, may well be used as benchmark solutions for other approximate solution procedures. Further approximations by selecting different trial functions, which may enlarge the present investigation also to the other boundary conditions, are possible. In addition, even one confines oneself to the trial functions used herein, many other closed-form solutions to direct problem of clamped-free column buckling seem to be at ease. This contribution may also be interpreted as the first step towards the closed-form solutions of eigenvalue problems of structural elements in closedform, which may be used in their monitoring and identification.

\section{References}

[1] Elishakoff, I., Eigenvalues of Inhomogeneous Structures: Unusual Closed-Form Solutions, CRC Press, Boca Raton, 2005.

[2] Antman, S.S., The theory of rods, pp.641-703 of Linear Theories of Elasticity and Thermoelasticity, Truesdell C. (ed.), Berlin, Springer, 1973.

[3] Love, A.E.H., A Treatise on the Mathematical Theory of Elasticity, Cambridge, at the University press, $4^{\text {th }}$ edition, 1927.

[4] Timoshenko, S., History of Strength of Materials, McGraw-Hill, New York, 1953.

[5] Oldfather, W.A., Ellis C.A., Brown D.M., Leonhard Euler's Elastic Curves. Isis, 20(1), $72-160,1933$.

[6] Euler, L., Sur la force des callones. Memories de L'Academie des Sciences et BellesLetteres (Berlin) 13, 252-282, 1759. (in French)

[7] Engesser, F., Ueber Krickfestigkeit gerader Staebe. Zeitschrift Architekten und Ingineure in Hannover 35, 455 (1899). (in German).

[8] Dinnik, A.N., Design of columns of varying cross-section, Transactions of the ASME, Applied Mechanics, 1929. 
[9] Duncan, N.J., Galerkin's method in mechanics and differential equations. Aeronautical Research Committee Reports and Memoranda, No. 1798, 1937.

[10] Elishakoff, I., Pellegrini, F., Exact and effective approximate solutions of some divergent type non-conservative problems. Journal of Sound and Vibration, 114, 144-148, 1987.

[11] Elishakoff, I., Pellegrini, F., Application of Bessel and Lommel functions and undetermined multiplier Galerkin method version for instability of non-uniform column. Journal of Sound and Vibration, 115, 182-186, 1987

[12] Elishakoff, I., Pellegrini, F., Exact solution for buckling of some divergence type nonconservative systems in terms of Bessel and Lommel functions. Computer Methods in Applied Mechanics and Engineering, 66, 107-119, 1988

[13] Elishakoff, I., Inverse buckling problem for inhomogeneous columns. International Journal of Solids and Structures, 38(3), 457-464, 2001.

[14] Elishakoff, I., Eisenberger, M., Delmas, A., Buckling and vibration of functionally graded columns sharing Duncan's mode shape, and new cases. Structures, 5, 170-174, 2016.

[15] Suresh S, Mortensen A. Fundamentals of functionally graded materials. London, UK: IOM Communications Limited, 1998.

[16] Mahamood, R.S., Akinlabi, E.T., Functionally Graded Materials, Springer, 2017.

[17] Ruta, G.C., Varano, V., Pignataro, M., Rizzi N.L., A beam model for the flexuraltorsional buckling of thin-walled memberse with some applications. Thin-Walled Structures, 46, 816,822, 2008.

[18] Ruta, G., Pignataro, M., Rizzi, N., A direct one-dimensional beam model for the flexuraltorsional buckling of thin-walled beams. Journal of Mechanics of Materials and Structures, 1(8), 1479-1496, 2006.

[19] Tatone, A., Rizzi, N., A one-dimensional model for thin-walled beams, pp. 312-320 in Trends in applications of mathematics to mechanics, edited by W. ed. Schneider et al., Longman, Avon, 1991.

[20] Gupta, R.K., Gunda, J.B., Janardhan, G.R., Rao, G.V., Post-buckling analysis of composite beams: Simple and accurate closed-form expressions. Composite Structures, 92, 1947-1956, 2010.

[21] Mercan, K., Civalek, O., Comparison of Small Scale Effect Theories for Buckling Analysis of Nanobeam. International Journal of Engineering and Applied Sciences, 9(3), 87-97, 2016.

[22] Abbondanza, D., Battista, D., Morabito, F., Pallante, C., Barretta, R., Luciano, R., de Sciarra, F.M., Ruta, G., Modulated linear dynamics of nanobeams accounting for higher gradient effects. International Journal of Engineering and Applied Sciences, 8(2), 1-20, 2016. 
[23] He, J.H., A new approach to non-linear partial differential equations. Communications in Nonlinear Science and Numerical Simulation, 2, 230-235, 1997.

[24] He, J.H., Variational iteration method for delay differential equations, Communications in Nonlinear Science and Numerical Simulation, 2, 235-236, 1997.

[25] He, J.H., Variational iteration method a kind of non-linear analytical technique: some examples. International Journal of Nonlinear Mechanics, 34, 699-708, 1999.

[26] He, J.H., Variational iteration method some recent results and new interpretations. Journal of Computational and Applied Mathematics, 207, 3-17, 2007.

[27] He, J.H., Wu, X.H., Variational iteration method: new development and applications. Computers and Mathematics with Applications, 54,881-894, 2007.

[28] Turkyilmazoglu, M., An optimal variational iteration method. Applied Mathematics Letters, 24(5), 762-765, 2011.

[29] Yilmaz, E., Inc, M., Numerical simulation of the squeezing flow between two infinite plates by means of the modified variational iteration method with an auxiliary parameter. Nonlinear Science Letters A, 1, 297-306, 2010.

[30] Hosseini, M.M., Mohyud-Din, S.T., Ghaneai H., Usman, M., Auxiliary parameter in the variational iteration method and its optimal determination. International Journal of Nonlinear Sciences and Numerical Simulation, 11(7), 495-502, 2010.

[31] Herişanu, N., Marinca, V., A modified variational iteration method for strongly nonlinear problems. Nonlinear Science Letters A, 1, 183-192, 2010.

[32] Altintan, D., Ugur, O., Generalisation of the Lagrange multipliers for variational iterations applied to systems of differential equations. Mathematical and Computer Modelling. 54, 2040-2050, 2011.

[33] He, J.H., Notes on the optimal variational iteration method. Applied Mathematics Letters. 25(10), 1579-1581, 2012.

[34] Coskun, S.B., Atay, M.T., Determination of critical buckling load for elastic columns of constant and variable cross-sections using variational iteration method. Computers and Mathematics with Applications, 58, 2260,2266, 2009.

[35] Chen, Y., Zhang, J., Zhang, Z., Flapwise bending vibration of rotating tapered beams using variational iteration method. Journal of Vibration and Control, 22(15), 3384$3395,2016$.

[36] Eroglu, U., Large deflection analysis of planar curved beams made of functionally graded materials using variational iterational method. Composite Structures, 136, 204 216, 2016.

[37] Yun-dong, L., Yi-ren, Y., Vibration analysis of conveying fluid pipe via He's variational iteration method. Applied Mathematical Modelling, 43, 409,420. 
[38] Eroglu, U., Tufekci, E., Small-Amplitude free vibrations of straight beams subjected to large displacements and rotation. Applied Mathematical Modelling, 53, 223-241, 2018.

[39] Budiansky, B., Theory of buckling and postbuckling behavior of elastic structures, pp. 165 in Advances in applied mechanics, 14, Academic Press, New York, 1974.

[40] Timoshenko, S.P., Gere, J.M., Theory of Elastic Stability. McGraw-Hill, New York, 1961.

[41] Pease, M.C., Methods of Matrix Algebra. Academic Press, New York, 1965.

[42] Tufekci, E., Arpaci, A., Exact solution of in-plane vibrations of circular arches with account taken of axial extension, transverse shear and rotatory inertia effects. Journal of Sound and Vibration 209 (5):845-56, 1998.

[43] Tufekci, E., Arpac1, A., Analytical solutions of in-plane static problems for non-uniform curved beams including axial and shear deformations. Structural Engineering and Mechanics, 22 (2):131-50, 2006.

[44] Yildirim, V., Several Stress Resultant and Deflection Formulas for Euler-Bernoulli Beams under Concentrated and Generalized Power/Sinusoidal Distributed Loads, International Journal of Engineering and Applied Sciences, 10(2), 35-63, 2018.

[45] Wei, D.J., Xani S.X., Zhang, Z.P., Li, X.F., Critical load for buckling of non-prismatic columns under self-weight and tip force. Mechanics Research Communications, 37, 554-558- 2010.

[46] Wang, C.M., Wang, C.Y., Reddy, J.N., Exact Solutions for Buckling of Structural Members. CRC Press, Boca Raton, 2005. 